\title{
ON SURFACES IN $\mathbf{R}^{4}$
}

\author{
WALTER SEAMAN
}

\begin{abstract}
We provide answers (Theorem $C$ ) to some questions concerning surfaces in $\mathbf{R}^{4}$ and maps into the quadric $Q_{2}$ raised by $\mathrm{D}$. Hoffman and $\mathbf{R}$. Osserman.
\end{abstract}

Let $S$ be an oriented surface immersed in $\mathbf{R}^{4}$. The Gauss map of $S$ is the map $G$ of $S$ into $G(2,4)$, the Grassmannian of oriented two-planes in $\mathbf{R}^{4}$, given by $G(p)=T_{p} S$. $G(2,4)$ can be identified with $Q_{2}$, the complex quadric in $\mathrm{C} P^{3}$, and in turn $Q_{2}$ is biholomorhic to $\mathbf{C} P^{1} \times \mathbf{C} P^{1}$. If we give $\mathbf{C} P^{3}$ the Fubini-Study metric of constant holomorphic sectional curvature 2 , then the induced metric on $Q_{2}$ is given by

$$
2\left|d w_{1}\right|^{2} /\left(1+\left|w_{1}\right|^{2}\right)^{2}+2\left|d w_{2}\right|^{2} /\left(1+\left|w_{2}\right|^{2}\right)^{2}
$$

where $\left(w_{1}, w_{2}\right)$ are coordinates on $\mathbf{C} \times \mathbf{C}$, viewed as local coordinates on $\mathbf{C} P^{1} \times \mathbf{C} P^{1}$ [1]. The metric $2|d w|^{2} /\left(1+|w|^{2}\right)^{2}$ is the metric on $\mathbf{C}$ induced by the map of $\mathbf{C}$ onto $S^{2}(1 / \sqrt{2}) \subset \mathbf{R}^{3}$ given by $w \mapsto \sigma^{-1}(\sqrt{2} w)$, where $\sigma^{-1}$ is inverse stereographic projection (with the sphere sitting on the $x y$-plane). Thus, $Q_{2}$ is isometric to $S^{2}(1 / \sqrt{2}) \times$ $S^{2}(1 / \sqrt{2})$. In particular, if $z$ is a local conformal parameter on $S$, then any map $G$ of $S$ into $Q_{2}$ splits into a pair of maps $G(z)=\left(f_{1}(z), f_{2}(z)\right)$, where $w_{i}=f_{i}(z)$ as above. Now define the following quantities on $S$ for $i=1,2$ :

$$
F_{i}:=\frac{f_{i \bar{z}}}{1+\left|f_{i}\right|^{2}}, \quad T_{i}(z)=\left[\frac{\left(f_{i}\right)_{z \bar{z}}}{\left(f_{i}\right)_{\bar{z}}}-\frac{2 \bar{f}_{i} f_{i z}}{1+\left|f_{i}\right|^{2}}\right]_{\bar{z}} \text { where } f_{i \bar{z}} \neq 0
$$

with the usual $z$ and $\bar{z}$ derivative notation. The following results are from $[1,2]$.

THEOREM A. For the Gauss map $G$ of an oriented surface $S$ immersed in $\mathbf{R}^{4}$, write $G=\left(f_{1}(z), f_{2}(z)\right)$ as above. Then we necessarily have

$$
\left|F_{1}\right| \equiv\left|F_{2}\right| \text {, }
$$

and

$$
\operatorname{Im}\left\{T_{1}+T_{2}\right\} \equiv 0 .
$$

THEOREM B. Let $S_{0}$ be a simply connected Riemann surface (here and subsequently), let $G=\left(f_{1}(z), f_{2}(z)\right)$ be some map of $S_{0}$ into $Q_{2}$, and define $F_{i}$ and $T_{i}$ as before, where $z$ is a conformal parameter on $S_{0}$.

(i) If $F_{1}=F_{2} \equiv 0$, then $G$ is the Gauss map of a minimal surface in $\mathbf{R}^{4}$, provided $S_{0}$ is not compact.

Received by the editors April 19, 1984.

1980 Mathematics Subject Classification. Primary 53A05; Secondary 53A10. 
(ii) If $F_{1}, F_{2}$ are never zero, then $G$ is the Gauss map of a surface $S$ in $\mathbf{R}^{4}$ given by a conformal immersion of $S_{0}$ if and only if

$$
\left|F_{1}\right| \equiv\left|F_{2}\right|
$$

and

$$
\operatorname{Im}\left\{T_{1}+T_{2}\right\} \equiv 0
$$

Furthermore, in this case $S$ is uniquely determined up to translation and homothety of $\mathbf{R}^{4}$.

Let $\left(1^{\prime}\right)$ denote the condition that $F_{1}, F_{2}$ are never zero (i.e., $f_{1 \bar{z}}$ and $f_{2 \bar{z}}$ are never zero) and $\left|F_{1}\right| \equiv\left|F_{2}\right|$. A special class of maps which satisfy (2) are harmonic maps, i.e., those $f(z)$ 's such that

$$
L(f):=f_{z \bar{z}}-2 \bar{f} f_{z} f_{z} /\left(1+|f|^{2}\right)=0 .
$$

In particular, if

$$
L\left(f_{i}\right) \equiv 0, \quad i=1,2,
$$

then (2) is automatically satisfied. Condition (3) is simply that the map $G: S_{0} \rightarrow Q_{2}$ is harmonic. A theorem of Ruh and Vilms [4] asserts that the Gauss map of a submanifold of $\mathbf{R}^{n}$ is harmonic if and only if the submanifold has parallel mean curvature. Combining this with Theorem $\mathrm{B}$ we now have the following observation:

A map $G: S_{0} \rightarrow Q_{2}$ is the Gauss map of a conformal immersion with parallel (nonzero) mean curvature in $\mathbf{R}^{4}$ if and only if $\left(1^{\prime}\right)$ and (3) hold.

Finally, an interesting subclass of surfaces of parallel mean curvature in $\mathbf{R}^{4}$ are minimal surfaces in some $S^{3}(r)$. Hoffman and Osserman also prove the following

Proposition. A map $G: S_{0} \rightarrow Q_{2}$ is the Gauss map of a conformal minimal immersion of $S_{0}$ into some $S^{3}(r)$ (viewed as sitting in $\mathbf{R}^{4}$ ) if and only if (1) and (3) are satisfied, as well as the following

$$
f_{1 z} / f_{1 \bar{z}}=f_{2 z} / f_{2 \bar{z}}
$$

In view of these results, the following questions present themselves [1, 2]: Given a map from $S_{0}$ into $S^{2}(1 / \sqrt{2})$, represented locally by $f_{1}(z)$ as above, does there exist a map from $S_{0}$ into $S^{2}(1 / \sqrt{2})$, represented by $f_{2}(z)$, such that the pair $\left(f_{1}(z), f_{2}(z)\right)$ satisfies

Q1. ( $\left.i^{\prime}\right)$ and (2)? Suppose $f_{1}$ satisfies $L\left(f_{1}\right)=0$. Does there exist $f_{2}(z)$ such that the pair $\left(f_{1}, f_{2}\right)$ satisfies

Q2. (1') and (3), or

Q3. (1'), (3) and (4)?

An affirmative answer to Q1 (Q2) would mean that the pair $\left(f_{1}, f_{2}\right)$ is the Gauss map of a conformal immersion (with parallel nonzero mean curvature) of $S_{0}$ in $\mathbf{R}^{4}$, while an affirmative answer to Q3 would mean that the pair $\left(f_{1}, f_{2}\right)$ is the Gauss map of a conformal minimal immersion of $S_{0}$ into some $S^{3}(r)$ (viewed as sitting in $\left.\mathbf{R}^{4}\right)$. 
We answer Q2 and Q3 affirmatively in Theorem C. While this provides an affirmative answer to Q1 under the special assumption of $(3)(\Rightarrow(2))$, we do not know the answer to $\mathrm{Q} 1$ in general.

THEOREM C. Given a map from $S_{0}$, not conformally equivalent to $S^{2}$, into $S^{2}(1 / \sqrt{2})$, written as $f_{1}(z)$ as above, such that $f_{1 z}$ is never zero, and $L\left(f_{1}\right)=0$, there exists a one-parameter family of maps of $S_{0}$ into $S^{2}(1 / \sqrt{2})$, written as $f_{\theta}(z)$, such that the pair $\left(f_{1}, f_{\theta}\right)$ satisfies (1) and (3). Furthermore, there is a unique $\theta_{0}$ such that the pair $\left(f_{1}, f_{\theta_{0}}\right)$ also satisfies (4). If $S_{0}$ is conformally equivalent to $S^{2}, f_{2}=f_{1}$ is the only possibility for even (1) and (3).

REMARK. The idea of the proof is to regard $f_{1}$ as the Gauss map of a surface $S$ of constant (nonzero) mean curvature in $\mathbf{R}^{3}$. The $f_{\theta}$ 's are the Gauss maps of the associated family $S_{\theta}, 0 \leqslant \theta \leqslant 2 \pi$, to $S$. It turns out that condition (4) is then satisfied exactly for the surface $S_{\pi}$ :

Proof of Theorem C. We regard $f_{1}(z)$ as the representation of a map of $S_{0}$ with $S^{2}(1)$ as follows: Let $\sigma\left(\sigma^{\prime}\right)$ be stereographic projection of $S^{2}(1 / \sqrt{2})\left(S^{2}(1)\right)$ onto C, and consider the transformation $\mathbf{C} \stackrel{\phi}{\rightarrow} \mathbf{C}$ by

$$
\phi(w)=\frac{1}{2}\left(\sigma^{\prime}\left(\sqrt{2}\left(\sigma^{-1}(\sqrt{2} w)\right)\right)\right) .
$$

$\phi$ is just the identity map on $\mathbf{C}$, so $\phi\left(f_{1}\right)=f_{1^{\prime}}$. Replacing $f_{1}$ by $\tilde{f}_{1}=\sqrt{2}\left(\sigma^{-1}\left(\sqrt{2} f_{1}\right)\right)$ $\in S^{2}(1)$, and then representing $\tilde{f}_{1}$ by $\frac{1}{2} \sigma^{\prime}\left(\tilde{f}_{1}\right)$, we see that we may regard $f_{1}$ as a map into $\mathbf{C} P^{1}$, with the metric $4|d w|^{2} /\left(1+|w|^{2}\right)^{2}$ of constant curvature 1 . Thus it suffices to prove Theorem C with $S^{2}(1 / \sqrt{2})$ replaced by $S^{2}(1)$. Now the conditions $f_{1 z} \neq 0, L\left(f_{1}\right)=0$ mean that $f_{1}$ is a harmonic, nowhere anticonformal map of $S_{0}$ into $S^{2}=S^{2}(1)$. From Hoffman-Osserman [1] and Kenmotsu [3], this guarantees that $f_{1}$ is the Gauss map of a conformal immersion $X$ of $S_{0}$ into $\mathbf{R}^{3}$ with constant nonzero mean curvature. If we specify that $X\left(S_{0}\right)$ have constant mean curvature 1 , then this determines $\mathscr{S}_{0}=X\left(S_{0}\right)$ up to translation in $\mathbf{R}^{3}$. If $S^{0}$ is conformally equivalent to $S^{2}$, then $\mathscr{S}_{0}$ is the standard unit sphere, and any $f_{2}: S_{0} \rightarrow S^{2}(1)$ satisfying (1) and (3) must come from the same $X$ (up to translation of $\mathbf{R}^{3}$ ). For $S_{0}$ not conformally $S^{2}$, in the (global) isothermal parameter $z$, the metric induced on $S_{0}$ is $4 F_{0}|d z|^{2}$ [3], where we have relabelled $f_{1}$ as $f_{0}$. Now consider the associate family $\mathscr{S}_{\theta}$ [5] to $\mathscr{S}_{0}(0 \leqslant \theta<2 \pi)$. Then the Gauss maps $f_{\theta}$ of $\mathscr{S}_{\theta}$ satisfy (3), since each $\mathscr{S}_{0}$ has constant mean curvature. Since the metric on $S_{0}$ inherited from $\mathscr{S}_{\theta}$ is given by

$$
4\left|f_{\theta}-\right|^{2} /\left(1+\left|f_{\theta}\right|^{2}\right)^{2}|d z|^{2}
$$

and since $\mathscr{S}_{\theta}$ is isometric to $\mathscr{S}_{0}$, we also have condition (1) satisfied for the pair $\left(f_{0}, f_{\theta}\right)$. Let $\beta^{\theta}$ be the second fundamental form of $\mathscr{S}_{\theta}$. Then from formula 5.3 of [3], we have

$$
\frac{1}{F_{\theta}}\left\{\frac{\beta_{11}^{\theta}-\beta_{22}^{\theta}}{2}-i \beta_{12}^{\theta}\right\}=\frac{f_{\theta z}}{f_{\theta \bar{z}}} .
$$


From this we see that condition (4), in the presence of (1), is equivalent to

$$
\beta_{11}^{0}-\beta_{22}^{0}=\beta_{22}^{\theta}-\beta_{11}^{\theta}, \quad \beta_{12}^{0}=-\beta_{12}^{\theta} .
$$

Finally, since

$$
\begin{aligned}
& \beta_{11}^{\theta}=\cos \theta\left(\beta_{11}^{0}-F_{0}\right)+\sin \theta \beta_{12}^{0}+F_{0}, \\
& \beta_{22}^{\theta}=-\cos \theta\left(\beta_{11}^{0}-F_{0}\right)-\sin \theta \beta_{12}^{0}+F_{0}, \\
& \beta_{12}^{\theta}=\cos \theta \beta_{12}^{0}-\sin \theta\left(\beta_{11}^{0}-F_{0}\right)
\end{aligned}
$$

and $\beta_{11}^{0}+\beta_{22}^{0}=2 F_{0}[5]$, we see that condition (4) (cf. (6)) is equivalent to $\theta=\pi$. Q.E.D.

\section{REFERENCES}

1. D. Hoffman and R. Osserman, The Gauss map of surfaces in $\mathbf{R}^{n}$, J. Differential Geom. 18 (1983).

2. , The Gauss map of surfaces in $\mathbf{R}^{3}$ and $\mathbf{R}^{4}$ (to appear).

3. K. Kenmotsu, Weierstrass formula for surfaces of prescribed mean curvature, Math. Ann. 245 (1979), 89-99.

4. E. Ruh and J. Vilms, The tension field of the Gauss map, Trans. Amer. Math. Soc. 149 (1970), 569-573.

5. B. Lawson, Complete minimal surfaces in $S^{3}$, Ann. of Math. (2) 92 (1970), 335-374.

Department of Mathematics, University of Iowa, Iowa City, Iowa 52242 